\title{
Small Intestinal Submucosa for Patch Grafting after Plaque Incision in the Treatment of Peyronie's Disease
}

\author{
Eugene W. Lee, Alan W. Shindel, Steven B. Brandes \\ Department of Surgery, Division of Urology, Washington University School of Medicine, St. Louis, \\ Missouri, USA
}

\begin{abstract}
Objective: Report the results using porcine small intestinal submucosa (SIS) as a graft material in the surgical management of Peyronie's disease (PD).

Materials and Methods: We performed a retrospective chart review of men with PD who underwent surgical correction of the curvature by plaque " $\mathrm{H}$ " incision and patch grafting with 4-ply SIS (Cook, Bloomington, IN) by a single surgeon at our institution. Degree and direction of curvature, sexual function, and co-morbidities were assessed pre- and postoperatively.

Results: Thirteen patients were identified. Mean age was $57 \pm 8$, range 42-70 years. Median follow-up was 14 months, range 3-89 months. At presentation, all reported penile curvature. Also reported were difficulty with vaginal penetration (determined by question number 2 of the sexual encounter profile questionnaire - SEP2),

palpable plaque, hourglass deformity, difficulty with firmness, and difficulty with sustaining erection (determined by SEP3) in $77 \%, 69 \%, 77 \%, 62 \%$, and $46 \%$ of patients, respectively. Mean and median degrees of curvature of the primary deformity were 71 and 67.5 degrees, respectively. Three patients had secondary curves of less than 30 degrees in a different direction. Mean and median plaque size were 3.5 and $2.7 \mathrm{~cm}^{2}$, respectively. Seven patients had one graft and six patients had two grafts placed with a mean size of $15 \pm 0 \mathrm{~cm}^{2}$.

Conclusions: For the patient with PD, SIS grafting can achieve a functionally straight erection with durable results yet with relatively high rates of erectile dysfunction. SIS is a viable graft material for use in the surgical treatment of PD.
\end{abstract}

Key words: penis; Peyronie's disease; surgery; graft; small intestinal submucosa

Int Braz J Urol. 2008; 34: 191-7

\section{INTRODUCTION}

Peyronie's disease (PD) is a condition of plaque formation in the tunica albuginea of the corpora cavernosa with resultant curvature of the penis (1). The disease has two phases; an early acute phase associated with painful erections and progression of deformity, and a later chronic phase in which the pain ceases and the deformity is stabilized. Conservative medical therapy is the initial treatment of choice for patients with acute phase disease. A variety of medications has been utilized including vitamin $\mathrm{E}$, paraaminobenzoate potassium, tamoxifen, colchicine, and verapamil. In addition to oral therapy, intralesional 
injections and various forms of energy have been applied for the treatment of PD (2). Few medical therapies have been proven effective in large scale trials. Surgical correction is the treatment of choice when the deformity precludes intercourse, but should not be considered until the disease has reached its stable, chronic phase.

Surgical options for the management of PD can be divided into 3 types: procedures that shorten the convex, uninvolved side of the tunica albuginea, procedures that lengthen the concave, diseased side, and implantation of a penile prosthesis (3). Ellipsoid resection and closure of the tunica albuginea on the healthy convex side, as first described by Nesbit, was the previous standard for surgical care (4). Subsequently, successful variations including a Heineke-Mikulicz procedure and multiple parallel plications have been reported $(5,6)$. These procedures are limited by a shorter postoperative penile length that precludes their use in patients with large plaques, severe deformities, or small penises (3). For these patients, superior results may be obtained using tunical lengthening procedures where incision or excision of the tunical plaque is followed by interposition of a graft material to bridge the resulting defect in the tunica. Plaque incision utilizing autologous materials such as saphenous vein as patch grafts for the incised defect has been reported to result in high rates of patient satisfaction $(7,8)$. Drawbacks to autologous grafting are the increased morbidity of a secondary tissue harvest site and increased OR time required for tissue procurement and preparation.

Non-autologous "off the shelf" biomaterials are an attractive alternative to autologous tissue for patch grafting (3). At our institution we favor porcine small intestinal submucosa (Stratasis, Cook Urological, Spencer, IN), a xenographic biomaterial retaining matrix elements that support host cell migration and differentiation. There have been some preliminary reports describing the use of SIS in Peyronie's disease repair (9-12). We present our institution's experience with SIS grafts in 13 patients.

\section{MATERIALS AND METHODS}

Institutional review board approval was obtained for a retrospective chart review of men who underwent surgical correction of Peyronie's disease with plaque incision and patch grafting with 4-ply porcine small intestinal submucosa (Stratasis, Cook Urological, Spencer, IN) at our institution by a single surgeon between March 1999 and August 2006.

Thirteen patients were identified. Subjective difficulty with vaginal penetration, firmness, and sustaining erection as well as age, erectile dysfunction, use of pro-erectile medications, presence of palpable plaque, plaque location, hourglass deformity, foreshortened penis, degree and direction of curvature, flaccid and stretched penile length, and comorbidities ( hypertension, diabetes mellitus, Dupuytren's contracture, plantar fasciitis, lower urinary tract symptoms) were included in the preoperative analysis. Sexual function was determined by SEP2.

Technique - The plaque incision and grafting procedure was carried out as detailed in prior reports (9). Briefly, subcoronal degloving incision was used with subsequent degloving of the penis down to dartos fascia to expose the anterior lamella of Buck's fascia. The area of maximal curvature was identified via artificial erection (Penrose tourniquet placement at the base of the penis and continuous corporal infusion of sterile saline). The circumflex veins were suture ligated or cauterized using bipolar current. If the plaque was dorsally located, the neurovascular bundle on the dorsal surface was mobilized under loupe magnification down to the penoscrotal junction. A relaxing incision was made in the plaque at the point of maximum curvature in the shape of the letter " $\mathrm{H}$ ". A graft of 4 layers SIS that was oversized to be $120 \%$ of the size of the defect was hydrated in normal saline and sewn in using 5-0 PDS running sutures. Assessment of curvature was repeated. Persistent curvature was managed by a second $\mathrm{H}$-incision and SIS graft or by penile plication (by the 16-dot technique of Lue) (6). The dartos was closed with a 3-0 vicryl suture and the skin with 3-0 chromic followed by a compressive Coban dressing for 48 hours.

Follow-up data was collected by the operating surgeon at follow-up office visits. Postoperative penile curvature, length, potency, use of erectile dysfunction medications, pain, and palpable plaque were recorded. Subjective (by patient) and objective (by physician) cosmetic and functional outcomes were recorded. 


\section{RESULTS}

Patient demographics are shown in Table-1. Mean age was $57 \pm 8$ years (range $=42-70$ years). The median length of follow-up was 14 months (range 389 months). Table-2 lists presenting symptoms and sexual function. Ten patients $(77 \%)$ presented with difficulty invaginal penetration (as determined by the sexual encounter profile question 2 [SEP2]), 8 patients $(62 \%)$ had difficulty maintaining firmness, and 6 patients $(46 \%)$ had difficulty sustaining erection (as determined by the sexual encounter profile question 3 [SEP3]). Pre-operatively, 5 patients (38\%) were potent, 7 patients $(54 \%)$ were potent with phosphodiesterase-5 inhibitors, and 1 (8\%) was impotent, as determined by SEP 2 and 3 .

The plaque was palpable in 9 patients $(69 \%)$, and 10 patients $(77 \%)$ had hourglass deformity. Penile curvature was dorsal in 8 patients $(62 \%)$, ventral in 2

Table 1 - Patient demographics.

\begin{tabular}{|c|c|c|}
\hline Age (years) & $57 \pm 8$ & Range $42-70$ \\
\hline Median follow-up (mo) & 14 & \\
\hline Characteristics of curvature & & $\%$ \\
\hline Dorsal & 8 & (62) \\
\hline Ventral & 2 & (15) \\
\hline Left & 3 & (23) \\
\hline Secondary curve present & 3 & (23) \\
\hline Mean degree curvature & 71 & range 45 - 90 \\
\hline Median degree curvature & 68 & \\
\hline Characteristics of plaque & & $\%$ \\
\hline Proximal & 2 & (15) \\
\hline Mid-shaft & 9 & (69) \\
\hline Distal & 2 & $(15)$ \\
\hline Mean size $\left(\mathrm{cm}^{2}\right)$ & 3.5 & range $1-8$ \\
\hline Median size $\left(\mathrm{cm}^{2}\right)$ & 2.7 & \\
\hline Palpable plaque & 9 & (69) \\
\hline Waist deformity & 10 & (77) \\
\hline \multicolumn{3}{|l|}{ Number of grafts placed } \\
\hline 1 & 7 & (54) \\
\hline 2 & 6 & $(46)$ \\
\hline Mean graft size $\left(\mathrm{cm}^{2}\right)$ & $15 \pm 10$ & range $1.5-36$ \\
\hline Concomitant penile plication & 11 & $(85)$ \\
\hline
\end{tabular}

Table 2 - Presenting symptoms.

\begin{tabular}{lc}
\hline Difficulty with vaginal penetration & $10(77 \%)$ \\
Difficulty maintaining firmness & $8(62 \%)$ \\
Difficulty sustaining erection & $6(46 \%)$ \\
Potency (SEP 2/3) & \\
$\quad$ Without ED medication & $5(38 \%)$ \\
$\quad$ With ED medications & $7(54 \%)$ \\
Not potent & $1(8 \%)$ \\
\hline
\end{tabular}

$E D=$ erectile dysfunction; $S E P=$ sexual encounter profile questionnaire.

patients (15\%), and toward the left in 3 patients $(23 \%)$. Three patients $(23 \%)$ had secondary curves of less than 30 degrees in a different direction. The degree of primary curvature ranged from 45 to 90 degrees, with mean of 71 and median of 68 . Nine plaques $(69 \%)$ were located in the mid-shaft, with $2(15 \%)$ in the proximal and $2(15 \%)$ in the distal portion of the phallus. Mean and median plaque size were 3.5 and $2.7 \mathrm{~cm}^{2}$, (range $=1-8$ ) respectively.

At operation, 7 (54\%) patients had one graft and $6(46 \%)$ patients had 2 grafts placed. Mean size of graft was $15 \pm 10 \mathrm{~cm}^{2}$ (range $\left.=1.5-36\right)$. Eleven patients $(85 \%)$ required concomitant penile plication for residual curvature.

Postoperative results are listed in Table-3. As determined by combined stimulation test (intracaver-

Table 3 - Postoperative data.

\begin{tabular}{ll}
\hline $\begin{array}{l}\text { Residual bend } \\
\text { None }\end{array}$ & $7(54 \%)$ \\
Residual bend present & $6(46 \%)$ \\
5 degree & $3(23 \%)$ \\
10 degree & $1(8 \%)$ \\
15 degree & $2(15 \%)$ \\
Potency (SEP 2/3) & \\
Without ED medication & $4(31 \%)$ \\
With ED medications & $2(15 \%)$ \\
Not potent & $7(54 \%)$ \\
Waist deformity & $2(15 \%)$ \\
Recurrent plaque & $4(30 \%)$ \\
\hline ED = erectile dysfunction; SEP = sexual encounter profile \\
questionnaire.
\end{tabular}


nosal injection of alprostadil), seven patients (54\%) had a completely straight phallus and six (46\%) had residual bends of less than 15 degrees (three 5 degree, one 10 degree, two 15 degree). At follow-up, 4 patients $(31 \%)$ were potent, 2 patients $(15 \%)$ were potent on medication, and 7 patients (54\%) were impotent (as determined by SEP 2 and 3$)$. Two patients (15\%) had hourglass deformity and there was recurrent plaque in 4 patients $(30 \%)$.

\section{COMMENTS}

Plaque incision and grafting is the procedure of choice for the treatment of severe Peyronie's disease. This precludes intercourse when patients have large plaques, severe curvature, or a short phallus, which makes the loss of penile length with plication procedures unacceptable. Autologous grafts such as saphenous vein have been used as patch grafts with satisfactory results. However, non-autologous grafts have the advantage of not requiring a second harvest site, decreasing operative time and patient morbidity as well as preserving graft material, such as saphenous vein, should the patient require coronary artery bypass surgery in the future.

SIS is an extracellular matrix that is 80 to 100 $\mu \mathrm{m}$ thick and composed of mainly Type I collagen. The matrix retains angiogenic and other growth factors even after processing, and induces a rapid infiltration of native cells and neovascularization, acting as a scaffold for cell differentiation and maturation (13). A study using SIS in the fascia lata of dogs demonstrated that as early as 6 weeks after grafting, the SIS is completely replaced with a well vascularized connective tissue, a well organized collagen framework and fiber orientation identical to that of the original tissue (14). Furthermore, SIS grafting of the tunica albuginea in rabbits has shown no significant inflammatory response, corporeal fibrosis, or loss of cavernous smooth muscle content (15).

Knoll first described the successful use of SIS for the correction of Peyronie's disease in 12 patients, with good functional results and no complications or patient complaints at 11 months mean follow-up except for one recurrence requiring reoperation (9). All patients achieved potency both preoperatively and postoperatively, with one requiring intracavernous injection therapy. In a later report at 20 months mean follow-up with 97 patients, Knoll described only 6\% with residual curvature of less than ten degrees and $84 \%$ of patients retaining the same degree of potency as preoperative with no penile shortening, pain, infection, hematoma, or bulging of graft site (16).

In our series of 13 patients, all deformities were straightened either completely or with minimal residual curvature. Our results are similar to Knoll, being durable with no recurrent Peyronie's disease after 14 months median follow-up, although 4 patients $(30 \%)$ had recurrent plaque and $2(15 \%)$ had residual hourglass deformity. No patients experienced infection, bulging, or immunologic reaction at the graft site. One patient had a hematoma at the surgical site that resolved spontaneously without significant sequelae.

John et al. published a case report series of 4 patients with less encouraging results, with 3 of their 4 patients suffering recurrent curvature; however, the grafts used were oversized by only $10 \%$ rather than $20 \%$ (10). The single non-recurrence was only 20 degrees, with the others being 45, 80, and 90 degrees, suggesting that the $10 \%$ oversizing may not have been sufficient to compensate for graft contracture in the more severe bends. Additionally, one recurrent bend had been previously straightened with polytetrafluoroethylene mesh and another was grafted with 1-ply rather than 4-ply SIS, making it difficult to draw comparisons with our series.

Breyer et al. also reported a much higher recurrence (37\%) and complication rate (37\%) using SIS grafting in 19 PD patients after 15 months mean follow-up (11). Their study used 1-ply rather than 4ply SIS, which may explain their higher recurrence rate, as compared with our and Knoll's series (9) (both using 4-ply SIS). Also, many of Breyer's complications were minor, including hematoma (26\%) and infection (5\%). Furthermore, although $37 \%$ had recurrent curvature, only one required subsequent plication (5\%). We feel that 1-ply SIS is too thin and contracts more, and thus only 4-ply should be used for PD grafting. Further study is clearly needed.

Most recently, Kovac and Brown compared outcomes at 22 months mean follow-up after dermal, pericardial, and SIS grafting for PD in a 36 patient 
series (12). They reported better maintenance of preoperative length and rigidity in patients with SIS versus dermal or pericardial grafts as well as significant improvements in ED as determined by the Sexual Health Inventory for Men (SHIM) score (17 postoperative vs. 10.1 preoperative). This is comparable with Knoll, who reported $84 \%$ maintenance of potency, as well as Breyer, who reported no increase in ED as determined by the SHIM score.

In contrast, patients in our series had a relatively high rate of postoperative erectile dysfunction as determined by SEP 2 and 3. One patient was impotent preoperatively but a penile implant was not a covered benefit of his insurance, and he underwent grafting in order to straighten his penis in order to have intercourse with a vacuum device. Seven patients $(54 \%)$ originally potent with oral phophodiesterase-5 inhibitors suffered from ED post-operatively. Possible explanations for postoperative ED rates higher than prior reports on grafting are: the large size of grafts and number of grafts needed to correct severe (60-90 degree) curvatures, ventral position of plaque, our surgical technique, and the graft itself (not precluding venous leak). Preoperatively, one of the patients had a ventral plaque, which has been shown to have the greatest likelihood of postoperative venous leak (17). The other 6 patients had curvatures of $>60$ degrees, with four having 90 degree bends. Additionally, five of the patients required 2 grafts to repair the defect. Importantly, the use of various instruments (e.g, International Index of Erectile Function, SHIM score, SEP 2/3) among series to help assess ED may also contribute to differences in reported ED rates, as these instruments were not designed specifically for PD patients.

Our findings with respect to postoperative ED are similar to those of Leungwattanakij et al. who used processed cadaveric pericardium instead of SIS. Five out of 8 patients reported difficulty with erections due to venous leakage at 30 months mean follow-up (18). The authors postulated that patients with large plaque size, ventral plaque, and severe curvatures $(>60)$ may be more likely to have postoperative cavernosal insufficiency leading to ED.

In our study, concomitant penile plication was performed in 11 of 13 cases. In order to prevent the need for plication in addition to the incision and grafting procedure, we have since modified our surgical technique. The SIS graft is oversized by $130 \%$ instead of $120 \%$, and the $\mathrm{H}$ incision is broader and larger. Moreover, we have started to excise a small, linear segment of the plaque before placement of the graft, a technique that other authors have found to negate the need for a secondary plication (19).

Weaknesses of our study include a retrospective design, small sample size, and the use of subjective assessments of satisfaction and erectile function. Future studies on graft materials for the surgical correction of PD should utilize objective means of characterizing penile blood flow, such as power Doppler ultrasonography of the penis, and objective measures of rigidity and tumescence, such as Rigiscan ${ }^{\circledR}$ nocturnal penile tumescence.

\section{CONCLUSIONS}

The results of our experience using SIS in the repair of Peyronie's disease demonstrate its efficacy in achieving a functional, straight erection with durable results. The advantages of SIS include ease of use and favorable biochemical characteristics as well as ready availability and lack of need for native tissue harvest with its attendant morbidity. Our study showed a relatively higher rate of venous leak erectile dysfunction than previously described, particularly in men with greater penile curvature at baseline. Larger studies investigating the true rates of erectile dysfunction using SIS as well as comparisons with other materials are warranted.

\section{CONFLICT OF INTEREST}

Dr. Steven B. Brandes is from Pfizer Speaker's Bureau and is also a consultant and speaker for American Medical Systems.

\section{REFERENCES}

1. Hauck EW, Weidner W: François de la Peyronie and the disease named after him. Lancet. 2001;357: 204951. 
2. Ralph DJ, Minhas S: The management of Peyronie's disease. BJU Int. 2004; 93: 208-15.

3. Kendirci M, Hellstrom WJ: Critical analysis of surgery for Peyronie's disease. Curr Opin Urol. 2004; 14: 3818.

4. Nesbit RM: Congenital curvature of the phallus: report of three cases with description of corrective operation. J Urol. 1965; 93: 230-2.

5. Yachia D: Re: Corporeal plication for surgical correction of Peyronie's disease. J Urol. 1993; 149: 869.

6. Gholami SS, Lue TF: Correction of penile curvature using the 16-dot plication technique: a review of 132 patients. J Urol. 2002; 167: 2066-9.

7. Lue TF, El-Sakka AI: Venous patch graft for Peyronie's disease. Part I: technique. J Urol. 1998; 160: 2047-9.

8. Montorsi F, Salonia A, Maga T, Bua L, Guazzoni G, Barbieri L, et al.: Evidence based assessment of longterm results of plaque incision and vein grafting for Peyronie's disease. J Urol. 2000; 163: 1704-8.

9. Knoll LD: Use of porcine small intestinal submucosal graft in the surgical management of Peyronie's disease. Urology. 2001; 57: 753-7.

10. John T, Bandi G, Santucci R: Porcine small intestinal submucosa is not an ideal graft material for Peyronie's disease surgery. J Urol. 2006; 176: 1025-8; discussion 1029.

11. Breyer BN, Brant WO, Garcia MM, Bella AJ, Lue TF: Complications of porcine small intestine submucosa graft for Peyronie's disease. J Urol. 2007; 177: 58991.
12. Kovac JR, Brock GB: Surgical outcomes and patient satisfaction after dermal, pericardial, and small intestinal submucosal grafting for Peyronie's disease. J Sex Med. 2007; 4: 1500-8.

13. Voytik-Harbin SL, Brightman AO, Kraine MR, Waisner B, Badylak SF: Identification of extractable growth factors from small intestinal submucosa. J Cell Biochem. 1997; 67: 478-91.

14. Dejardin LM, Arnoczky SP, Clarke RB: Use of small intestinal submucosal implants for regeneration of large fascial defects: an experimental study in dogs. J Biomed Mater Res. 1999; 46: 203-11.

15. Monga M, Cosgrove D, Zupkas P, Jain A, Kasyan A, Wilkes N, et al.: Small intestinal submucosa as a tunica albuginea graft material. J Urol. 2002; 168: 1215-21.

16. Knoll LD: Use of small intestinal submucosa graft for the surgical management of Peyronie's disease. J Urol. 2007; 178: 2474-8; discussion 2478.

17. Kendirci M, Nowfar S, Gur S, Jabren GW, Sikka SC, Hellstrom WJ: The relationship between the type of penile abnormality and penile vascular status in patients with peyronie's disease. J Urol. 2005; 174: 632-5; discussion 635.

18. Leungwattanakij S, Bivalacqua TJ, Reddy S, Hellstrom WJ: Long-term follow-up on use of pericardial graft in the surgical management of Peyronie's disease. Int J Impot Res. 2001; 13: 183-6.

19. Personal communication, Dr. Gerald H Jordan, Director, Devine Center for Genitourinary Reconstruction, Norfolk, Virginia (USA).
Accepted after revision:

January 18, 2008

\footnotetext{
Correspondence address:

Dr. Stephen B. Brandes

Department of Surgery, Division of Urology

Washington University School of Medicine

4960 Children's Place

Box 8242, Saint Louis, MO, 63112, USA

Fax: + 1314 367-5016

E-mail: brandess@wustl.edu
} 


\section{EDITORIAL COMMENT}

As authors described in this paper, surgical approaches for the correction of PD can be divided into three basic categories; tunical plication, plaque excision (incision) and grafting procedures, and penile prosthesis implantation. Regarding to grafting materials, saphenous vein grafts are the most widely used with acceptable outcome in long-term. However, ideal "off the shelf" biomaterial is warranted because harvesting autologous material can cause the pain at donor site and longer operative time. Various off-theshelf materials including SIS are currently reported (1).

Authors reported on 13 patients with PD who treated with SIS grafting. Seven patients (54\%) had a completely straight phallus and six (46\%) had residual bend less than 15 degree after 14-month follow-up. Although only one patient had erectile dysfunction preoperatively, 7 patients were impotent postoperatively. From these results, SIS grafts would allow for satisfactory clinical results despite relatively high rate of erectile dysfunction.
However, long-term outcome of surgery for penile curvature is quite important to evaluate the durability of the procedure and treatment strategies. Unfortunately, longer follow-up with adequate number of patient treated with off-the-shelf material are still lacking. Additionally, since it is recognized that the majority of men with PD have vascular comorbidities that contribute to sexual dysfunction (2), surgery is not an exclusive factor that causes postoperative erectile dysfunction. Better understanding of the natural history of PD and long-term outcome of each surgical procedure are still needed.

\section{REFERENCES}

1. Kadioglu A, Sanli O, Akman T, Ersay A, Guven S, Mammadov F: Graft materials in Peyronie's disease surgery: a comprehensive review. J Sex Med. 2007; 4: 581-95.

2. Deveci S, Palese M, Parker M, Guhring P, Mulhall JP: Erectile function profiles in men with Peyronie's disease. J Urol. 2006; 175: 1807-11; discussion 1811.

Dr. Kimihiko Moriya

Department of Urology

Hokkaido University Graduate Sch of Med

Sapporo, Japan

E-mail:k-moriya@med.hokudai.ac.jp 